\title{
Optimization of Metal/Insulator/Metal structured emitter for spectrally controlled Metal/Semiconductor/Metal structured thermophotovoltaic cell
}

\author{
Han Tianyi ${ }^{1}$, Ryota Okino, Kazuma Isobe, Katsunori Hanamura ${ }^{1}$ \\ ${ }^{1}$ Department of Mechanical Engineering, Tokyo Institute of Technology, \\ Ookayama 2-21-1, Meguro-ku, Tokyo 152-8550, Japan
}

\begin{abstract}
A spectral emittance of Metal/Insulator/Metal (MIM) structured emitter was optimized for a spectrally controlled Metal/Semiconductor/Metal (MSM) structured thermophotovoltaic (TPV) cell using a Bayesian optimization method. Corresponding to the spectral absorptance of MSM cell around the bandgap wavelength of GaSb semiconductor, an emittance with an almost unity only around the bandgap could be achieved by selecting the adequate structure of MIM emitter.
\end{abstract}

Key Words: MIM emitter, MSM TPV cell, optimization

In recent years, more and more researchers focused on the thermophotovoltaic (TPV) system, which is mainly consist of heat source, emitter and TPV cell. A emitter receives thermal energy from the high temperature body (heat source). A TPV cell which absorbs the incident photons from the emitter and then directly converted it into electricity if the photon energy is higher than the bandgap of TPV cell. The photon which is lower than that of the bandgap of TPV cell can be converted into the waste heat and affect the performance of TPV cell. Thus, it requires to suppress long wavelength range of both emittance of the emitter and absorptance of the TPV cell.

In previous study, an Metal/Semiconductor/Metal (MSM) TPV cell was proposed by numerical calculation using the finite time difference (FDTD) method and it was also be manufactured successfully. Gold (Au) and gallium antimony (GaSb) are utilized as the materials of the metal and the thin semiconductor layer. By adding a Au-fishnet structure on the surface of the GaSb layer with the thickness of 100nm, the peak wavelength of the cell has transitioned to the region under the bandgap of Gasb which is $1.8 \mu \mathrm{m}$. And the absorptance of the MSM TPV cell reaches 1.0 at a wavelength of $1.55 \mu \mathrm{m}$. The radiant energy which can be absorbed is concentrated in the active wavelength range of GaSb cell (from around $0.8 \mu \mathrm{m}$ to $1.8 \mu \mathrm{m}$ ).

In this study, a Metal/Insulator/Metal (MIM) emitter which emits photons in a certain spectral ranges that matches with a MSM TPV cell, due to the emitted radiant energy can be generated into electricity only when the photon energy is higher than the bandgap of the semiconductor material utilized in the TPV cell. AMIM emitter is consist of the metal substrate, thin insulator layer on the surface of substrate and the metal island structure on the surface of insulator layer.

In order to further compare the effects of different materials on the radiation characteristics of the surface island structure, $\mathrm{W} / \mathrm{SiO} 2 / \mathrm{W}, \mathrm{Au} / \mathrm{Al} 2 \mathrm{O} 3 / \mathrm{Au}$ and $\mathrm{Ag} / \mathrm{Al} 2 \mathrm{O} 3 / \mathrm{Ag}$ emitters are simultaneously used and the size of the emitters are optimized by Bayesian optimization method. In the process of optimizing the geometric parameters of three different materials based on Bayesian optimization, each structure corresponds to three parameters width of the island " $\boldsymbol{\omega}$ ", height of the island " $\boldsymbol{h}$ " and thickness of insulator layer ' $d$ '. By changing the values of these parameters, the emittance of MIM emitter can be changed, thus affecting the value of the objective function.

In this research, three kinds of MIM emitter have been successfully designed and optimized for matching the MSM cell. The emittance of $\mathrm{W} / \mathrm{SiO} 2 / \mathrm{W}$ emitter is almost unity only around the bandgap of MSM cell. Meanwhile, the emittance of $\mathrm{Au} / \mathrm{Al} 2 \mathrm{O} 3 / \mathrm{Au}$ emitter and $\mathrm{Ag} / \mathrm{Al} 2 \mathrm{O} 3 / \mathrm{Ag}$ emitter have the same peak with the absorptance of MSM cell. The efficiency of TPV system is increasing and reach uniform while temperature is increasing. The $\mathrm{Au} / \mathrm{Al} 2 \mathrm{O} 3 / \mathrm{Au}$ emitter and $\mathrm{Ag} / \mathrm{Al} 2 \mathrm{O} 3 / \mathrm{Ag}$ emitter show a better efficiency than that of $\mathrm{W} / \mathrm{SiO} 2 / \mathrm{W}$ emitter, especially the $\mathrm{Au} / \mathrm{A} 2 \mathrm{O} 3 / \mathrm{Au}$ emitter has the maximum efficiency while the temperature is around $1300 \mathrm{~K}$. 